\title{
Review: oral anticoagulants plus antiplatelet agents reduce thromboembolism and all cause mortality for heart valve prostheses
} Massel D, Little SH. Risks and benefits of adding anti-platelet therapy to warfarin among patients with prosthetic heart
valves: a meta-analysis.J Am Coll Cardiol Feb 2001;37:569-78.

\section{QUESTION: In patients with mechanical prosthetic heart valves, are combined oral anticoagulants and antiplatelet treatment more efficacious than anticoagulants alone?}

\section{Data sources}

Studies were identified by searching Medline (1966 to November 1999) with the terms heart valve prosthesis, mechanical heart valve, thromboembolism, anticoagulant, antiplatelet, and haemorrhage. Bibliographies of studies, reviews, meta-analyses, and consensus statements were also checked.

\section{Study selection}

Full text and abstracts of randomised controlled trials were selected if patients with prosthetic heart valves were studied, oral anticoagulation plus antiplatelet agents were compared with oral anticoagulation alone, and objective methods were used to assess outcomes and adverse effects.

\section{Data extraction}

Data were extracted on study characteristics and quality; interventions, including type and duration of anticoagulation and antiplatelet agents; target international normalised ratio (INR) or prothrombin time ratio; and outcomes (all cause mortality, thromboembolism [VTE], and major haemorrhagic events).

\section{Main results}

10 RCTs (2199 patients) met the inclusion criteria. 6 studies evaluated dipyridamole, and 4 evaluated aspirin. All trials reported VTE events and all cause mortality; 2 trials did not report haemorrhagic events. The combination of antiplatelet agents and oral anticoagulants reduced the incidence of VTE events $(\mathrm{p}<0.001)$ and all cause mortality $(\mathrm{p}<0.001)$, while increasing the rate of major haemorrhagic events $(\mathrm{p}=0.03)$ (table). Both aspirin and dipyridamole showed similar reductions in all cause mortality and VTE events. Because the recommended dose of aspirin has decreased over time, subgroup analyses were done for trials before and after 1990. Early trials showed that major bleeding events were increased (odds ratio [OR] 2.23, 95\% CI 1.28 to 3.87); later trials showed no difference (OR 0.88 , CI 0.37 to 2.16$)$.

\section{Conclusions}

Adding antiplatelet agents to oral anticoagulants decreases thromboembolic events and all cause mortality in patients with mechanical prosthetic heart valves. The increased incidence of major bleeding events with combined treatment has diminished in the current, lower dose era.

Source of funding: not stated.

For correspondence: Dr D Massel, London Health Sciences Center, Room 205, Colborne Building, Victoria Campus, 375 South Street, London, Ontario N6A 4G5, Canada. Fax +1519667 $6687 / 6648$.

Antiplatelet treatment added to oral anticoagulants (combined therapy) $v$ anticoagulants alone (single treatment) for patients with mechanical prosthetic heart valves (follow up I to $2.5 \mathrm{y}$ )*

\begin{tabular}{lllll} 
& \multicolumn{2}{c}{ Weighted event rates } & & \\
\cline { 2 - 4 } Outcomes & $\begin{array}{l}\text { Combined } \\
\text { treatment }\end{array}$ & $\begin{array}{l}\text { Single } \\
\text { treatment }\end{array}$ & RRR (95\% Cl) & NNT (Cl) \\
\cline { 1 - 2 } Thromboembolic events & $5.5 \%$ & $8.9 \%$ & $57 \%(38$ to 70$)$ & 30 (19 to 62$)$ \\
\hline All cause mortality & $5.8 \%$ & $9.5 \%$ & $49 \%(35$ to 67$)$ & 27 (18 to 35$)$ \\
\hline Haemorrhagic events & $6.4 \%$ & & RRI (Cl) & NNH (Cl) \\
\hline
\end{tabular}

*Abbreviations defined in glossary. Weighted event rates, RRI, and NNH and related Cls calculated from data in article.

\section{COMMENTARY}

A commonly followed scheme for prophylaxis of VTE events from prosthetic valves on the basis of task force recommendations ${ }^{1}$ and consideration of the evidence for valve type and location as they affect the incidence of VTE events is included in a review by Fuster and Verstraete. ${ }^{2}$

Before the addition of platelet inhibitors, VTE rates for mechanical valves remained substantial despite meticulous anticoagulant treatment. Several studies adding aspirin, 500 to $1000 \mathrm{mg}$ /day, or dipyridamole showed reductions in VTE events with increases in serious haemorrhages. Turpie $e t$ al showed that low dose aspirin (50 to $100 \mathrm{mg} / \mathrm{d}$ ) reduced VTE events without an increase in major haemorrhage. ${ }^{3}$ This finding was verified by Meschengieser $e t a l{ }^{4}$ who found that VTE events could be controlled by warfarin with lower target INR plus low dose aspirin.

By using extensive meta-analysis techniques, Massel and Little showed that addition of a platelet inhibitor to warfarin reduced the risk for both VTE events and all cause mortality, although the risk for bleeding was increased. They found further that dipyridamole and high dose aspirin used in the older studies probably increased bleeding more than low dose aspirin. ${ }^{34}$ The results of these studies combined with the different propensities of mechanical and tissue valves in mitral or aortic positions to embolise led to the current practice of combining anticoagulation treatment with warfarin and low dose aspirin. ${ }^{12}$

Jay S Meisner, MD, PhD Aung Hla, MD

Albert Einstein College of Medicine, Bronx, New York, USA

1 Stein PD, Alpert JS, Dalen JE, et al. Antithrombotic therapy in patients with mechanical and biological prosthetic heart valves. Chest 1998;114:602S-10S.

2 Fuster V, Verstraete M. Hemostasis, thrombosis, fibrinolysis, and cardiovascular disease. In Alexander R. Fuster V, King S, et al, editors. Hurst's the heart. 10th edition. New York: McGraw-Hill; 2001:1777-81.

3 Turpie AG, Gent M, Laupacis A, et al. A comparison of aspirin with placebo in patients treated with warfarin after heart-valve replacement. N Engl J Med 1993;329:524-9.

4 Meschengieser SS, Fondevila CG, Frontroth J, et al. Low-intensity oral anticoagulation plus low-dose aspirin versus high-intensity oral anticoagulation alone: a randomized trial in patients with mechanical prosthetic heart valves. J Thorac Cardiovasc Surg 1997;113:910-6. 